09,01

\title{
Оптические эффекты в магнитных гиперболических метаматериалах
}

\author{
(C) А.Р. Помозов ${ }^{1}$, И.А. Колмычек ${ }^{1}$, Е.А. Ганьшина ${ }^{1}$, О.Ю. Волкова ${ }^{2}$, \\ А.П. Леонтьев ${ }^{2}$, К.С. Напольский ${ }^{2,3}$, Т.В. Мурзина ${ }^{1, \uparrow}$ \\ ${ }^{1}$ Московский государственный университет им. М.В. Ломоносова (физический фракультет), \\ Москва, Россия \\ ${ }^{2}$ Московский государственный университет им. М.В. Ломоносова (факультет наук о материалах), \\ Москва, Россия \\ ${ }^{3}$ Московский государственный университет им. М.В. Ломоносова (химический фракультет), \\ Москва, Россия \\ I E-mail: murzina@mail.ru
}

\begin{abstract}
Представлены результаты синтеза, экспериментального исследования и численного моделирования оптических и магнитооптических свойств массивов золотых и двухслойных $\mathrm{Au} / \mathrm{Ni}$ наностержней в матрице пористого анодного оксида алюминия. Показано наличие двух особенностей в спектрах таких структур в окрестности длин волн 520 и $700-800 \mathrm{~nm}$. Для наностержней $\mathrm{Au} / \mathrm{Ni}$ в области длинноволнового резонанса наблюдается значительная модуляция и усиление знакопеременного магнитооптического интенсивностного эффекта. Экспериментально наблюдаемые особенности согласуются с результатами численного моделирования.
\end{abstract}

Работа выполнена при поддержке грантов РФФИ № 18-02-00830_а и Президента РФ МК-5704.2018.2.

DOI: 10.21883/FTT.2018.11.46667.09NN

\section{1. Введение}

Гиперболическими метаматериалами называют композитные искусственные наноструктурированные материалы, в которых поперечная и продольная составляющие тензора диэлектрической и/или магнитной проницаемостей имеют противоположные знаки $[1,2]$. Такие среды сильно анизотропны - в одном направлении они могут вести себя как металл, а в другом - как диэлектрик. Гиперболический закон дисперсии приводит к появлению в оптических спектрах таких структур особенностей, связанных с возникновением полюса эффективной диэлектрической проницаемости (Epsilon Near Pole, ENP) или сменой знака действительной части эффективной диэлектрической проницаемости при прохождении ее через нулевое значение (Epsilon Near Zero, ENZ) для различных компонент [3]. Кроме того, в гиперболических средах к настоящему времени экспериментально обнаружены и теоретически объяснены такие эффекты как отрицательная рефракция [4,5], усиление спонтанного излучения [6,7] и рамановского рассеяния [8], преобразование ближнего поля объекта (излучаемого или рассеиваемого) в изображение в дальнем поле [9]. Оптика гиперболических сред сейчас находится на пике популярности исследований, что связано с широкими возможностями их применения в нанофотонике, биосенсорике $[10,11]$, построении изображений [12] и создании волноводов, обладающих рядом уникальных свойств [13,14].

Одной из простейших реализаций такой среды является массив наностержней металла (обычно золота или серебра) в диэлектрической матрице $[15,16]$. В таком случае закон дисперсии имеет вид:

$$
\frac{k_{x}^{2}+k_{y}^{2}}{\varepsilon_{\|}}+\frac{k_{z}^{2}}{\varepsilon_{\perp}}=\left(\frac{\omega}{c}\right)^{2},
$$

где ось $z$ направлена вдоль оси стержней, $\varepsilon_{\|}=\varepsilon_{z z}$, $\varepsilon_{\perp}=\varepsilon_{x x}=\varepsilon_{y y}$ могут быть вычислены в модели эффективной среды. Система координат приведена на рис. 1.

Подобные структуры, а именно, массивы золотых наностержней, полученные методом темплатного электроосаждения в матрицу пористого анодного оксида алюминия, уже исследовались в ряде работ $[17,18]$. Обнаружено, что локальный поверхностный плазмон, возбуждаемый в направлении, перпендикулярном длинной оси стержней, соответствует полюсу (ENP) действительной части диэлектрической проницаемости $\varepsilon_{\perp}$. Второй резонанс, зарегистрированный в спектре экстинкции, соответствует коллективным продольным колебаниям электронов в наностержнях и точке ENZ для компоненты диэлектрической проницаемости вдоль их осей $\varepsilon_{\|}$.

Новым направлением в области оптики гиперболических метаматериалов является создание структур, в которых законом дисперсии можно управлять, в том числе переключать гиперболический закон дисперсии в эллиптический и обратно, путем приложения внешнего статического магнитного поля. Например, в работе [19] рассчитываются компоненты магнитной проницаемости мультислойной структуры диэлектрик/гиромагнитный железо-иттриевый гранат и показано, что включение магнитного поля меняет знак плоскостных компонент $\mu$, обратимо включая таким образом гиперболический режим дисперсии в гигагерцовом диапазоне частот.

Здесь также следует упомянуть, что существуют варианты гиперболических сред, где за счет резонансного 

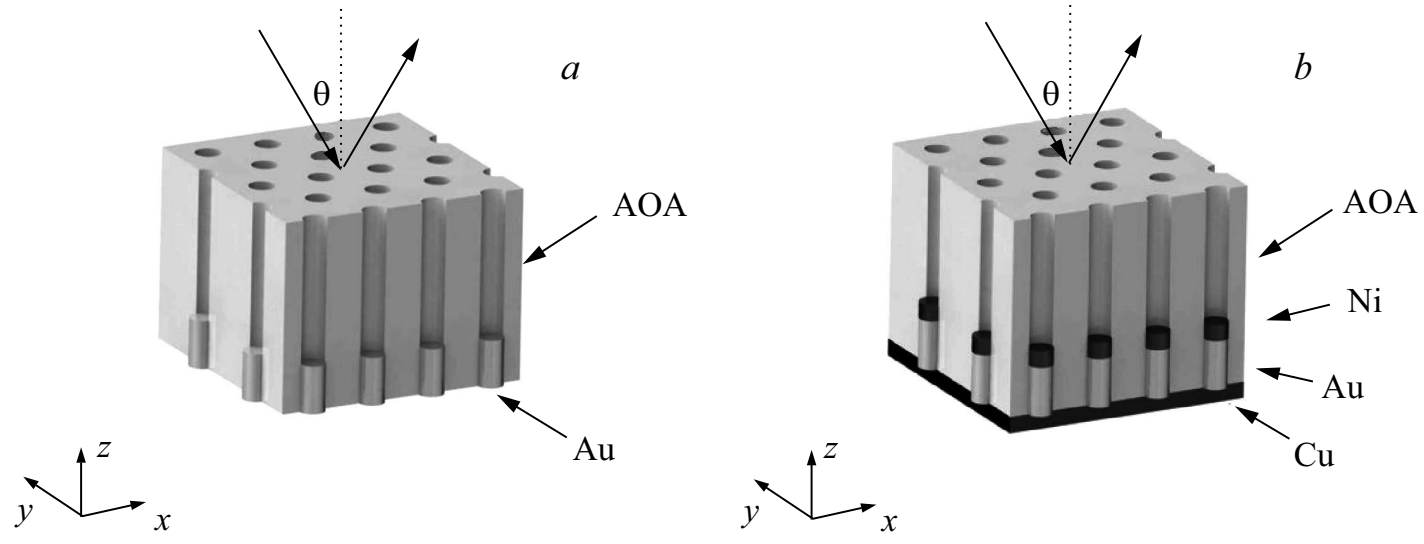

c
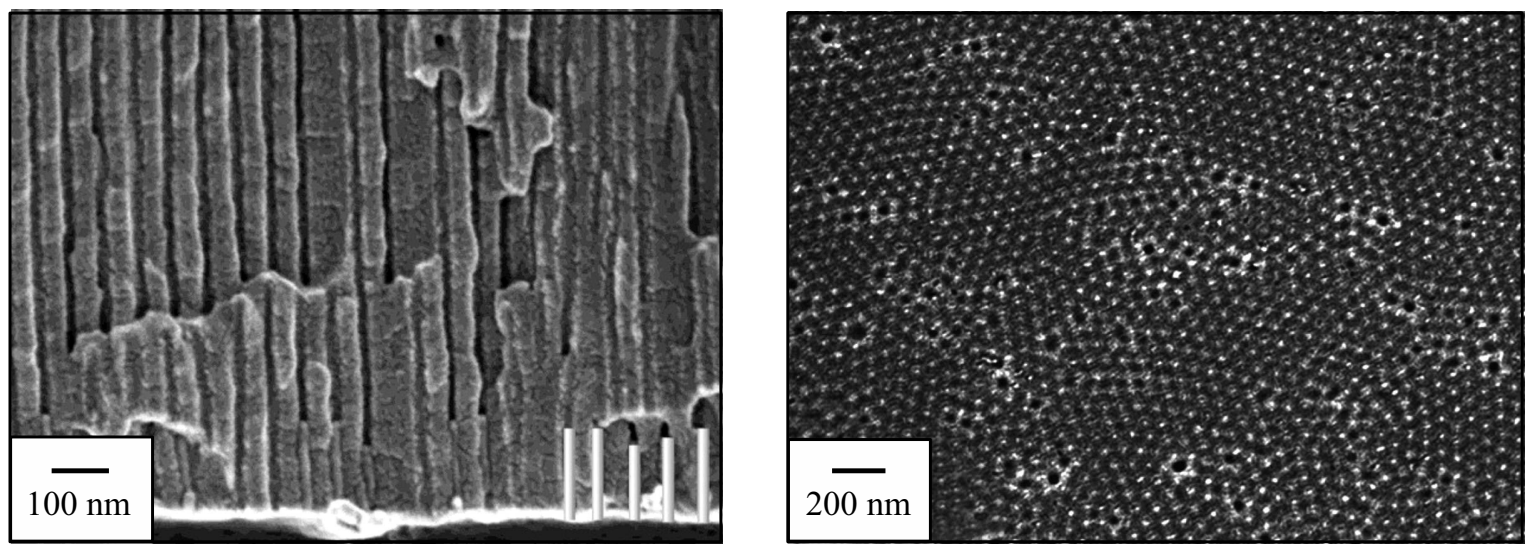

Рис. 1. Схемы образцов, содержащих наностержни $\mathrm{Au}(a)$ и $\mathrm{Au} / \mathrm{Ni}(b)$ в матрице анодного оксида алюминия. РЭМ-изображения поперечного скола $(c)$ и нижней поверхности $(d)$ образца с наностержнями Аu. На поперечном сколе приведено схематическое изображение наностержней.

возбуждения магнитного диполя меняется знак компонент магнитной проницаемости (в системе наностержней, изготовленных из материала с высоким показателем преломления [20]) или знаки компонент и $\varepsilon$, и $\mu$. (в мультислойных структурах типа „,fishnet“ [21]). Зачастую в литературе именно к таким средам применяют термин „магнитные гиперболические метаматериалы“. В настоящей работе представлены композитные магнитные гиперболические метаматериалы, работающие по другой „схеме“. Наличие статической намагниченности в гиротропной среде меняет ее диэлектрическую проницаемость, а следовательно, эффективную диэлектрическую проницаемость всей структуры, что дает возможность управления законом дисперсии путем приложения внешнего магнитного поля. Массивы никелевых наностержней в диэлектрической матрице уже исследовались в работах [17,22], однако в никеле добротность плазмонных резонансов относительно низка. Поэтому оптимальный дизайн структуры - композитные наностержни, состоящие из золота и никеля. В золоте возможно эффективное возбуждение плазмонов, а никель необходим для магнитооптической активности структуры. Следует также отметить, что магнитоплазмонные структуры также уже исследовались в ряде работ [23], где наблюдалось значительное усиление магнитооптических эффектов при возбуждении различных типов резонансов - локальных поверхностных плазмонов [24], бегущих плазмон-поляритонов [25], а также решеточных (дифракционных) плазмонов и волноводных мод [26], однако указанные эффекты наблюдались не в режиме гиперболической дисперсии.

В настоящей работе представлены результаты исследования оптического отклика массивов золотых и композитных (золото/никель) наностержней в режиме гиперболической дисперсии. Экспериментальные данные сопоставлены с результатами расчетов в рамках модели эффективной среды.

\section{2. Образцы}

Массивы металлических наностержней были получены методом темплатного электроосаждения с использованием в качестве матриц пористых пленок анодного оксида алюминия (AОА) толщиной $50 \mu \mathrm{m}$. При формировании слоя АОА применяли двухстадийное анодирование высокочистого алюминия $(99.99 \%)$ в $0.3 \mathrm{M}$ рас- 
творе $\mathrm{H}_{2} \mathrm{SO}_{4}$. Согласно статистическому анализу данных растровой электронной микроскопии (РЭМ), среднее расстояние между центрами пор шаблона АОА составляет около $64 \mathrm{~nm}$, диаметр пор $28 \mathrm{~nm}$, что соответствует пористости около $17 \%$. После химического растворения оставшегося после анодирования алюминия и барьерного слоя оксида на нижней стороне матрицы AOA методом магнетронного напыления формировали слой серебра или меди толщиной $150 \mathrm{~nm}$, служивший токосъемником при электроосаждении металла в поры. Электрокристаллизацию металлов проводили в трехэлектродной электрохимической ячейке в потенциостатическом режиме при комнатной температуре. Наностержни золота были получены из электролита 04-3Г (Экомет, Россия), содержащего $0.05 \mathrm{M}\left[\mathrm{Au}(\mathrm{CN})_{2}\right]^{-}$, а сегменты $\mathrm{Ni}-$ из электролита, содержащего $0.7 \mathrm{M} \mathrm{Ni}_{2} \mathrm{SO}_{4}$ и $0.3 \mathrm{M} \mathrm{H}_{3} \mathrm{BO}_{3}$.

Перед проведением оптических измерений серебряный электрод с поверхности АОА был селективно растворен в разбавленной $\mathrm{HNO}_{3}$. Схемы полученных структур и РЭМ-изображения нижней поверхности, а также скола образца с наностержнями Аu приведены на рис. 1.

\section{3. Экспериментальные установки и методики расчета}

Измерение спектров экваториального магнитооптического эффекта Керра (МОЭК) проводили при приложении к образцу насыщающего магнитного поля около $2 \mathrm{kG}$. Угол падения света на образец составлял $45^{\circ}$. Измеряемой величиной является магнитный контраст, вычисляемый как $\rho=(R(H)-R(-H)) /(R(H)+R(-H))$, где $R(H)$ и $R(-H)$ - интенсивности отраженного от образца излучения, измеренные при противоположных направлениях магнитного поля [27].

Расчеты компонент диэлектрической проницаемости проводились в программном пакете Wolfram Mathematica в рамках модели эффективной среды. Для композитных наностержней были учтены два слоя: оксид алюминия + золото и оксид алюминия + никель. Вначале были вычислены эффективные $\varepsilon_{\|}$и $\varepsilon_{\perp}$. для каждого из слоев, а затем были рассчитаны компоненты диэлектрической проницаемости всей структуры согласно уравнениям, приведенным в работе [4]. Диполь-дипольное взаимодействие наностержней было учтено аналогично работе [16]. Значения диэлектрических проницаемостей материалов были взяты из книги [28]. Расчеты спектров отражения и пропускания проводились методом матриц распространения.

\section{4. Результаты и обсуждение}

\section{1. Оптический отклик золотых наностержней}

На рис. 2 представлены экспериментальные частотноугловые спектры пропускания массивов золотых наностержней в матрице анодного оксида алюминия. В спек-

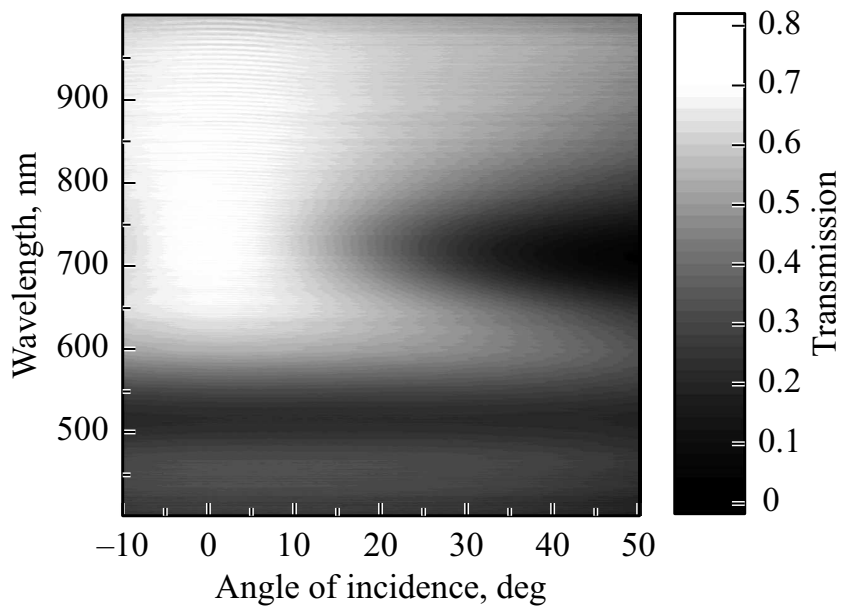

Pис. 2. Экспериментальный частотно-угловой спектр массива золотых наностержней с диаметром $28 \mathrm{~nm}$ и длиной $200 \mathrm{~nm}$. Пористость матрицы - 17\%.

трах хорошо видны минимумы на длинах волн 520 и $720 \mathrm{~nm}$, положение которых практически не изменяется с увеличением угла падения зондирующего излучения. Коротковолновый минимум соответствует возбуждению плазмонного резонанса в направлении, перпендикулярном стержню. Второй - возбуждению коллективных синфазных колебаний электронов вдоль стержней. Длинноволновый резонанс не возбуждается при нормальном падении света на образец, так как в таком случае отсутствует компонента поля накачки вдоль стержня. Добротность этого резонанса существенно возрастает при увеличении угла падения.

С помощью статистической обработки РЭМ-изображений скола и нижней поверхности образца были определены значения пористости $(17 \%)$ и длины $(200 \mathrm{~nm})$ наностержней $\mathrm{Au}$. C использованием этих параметров были рассчитаны спектры компонент диэлектрической проницаемости образца, представленные на рис. 3, a. Видно, что вблизи длины волны $530 \mathrm{~nm}$ компонента $\varepsilon_{\perp}$ (пунктирная линия) имеет полюс (ENP), а при длине волны $700 \mathrm{~nm}$ компонента $\varepsilon_{\|}$(штриховая линия) обращается в ноль (ENZ). Таким образом, в области длин волн 700-900 nm $\varepsilon_{\|}<0, \varepsilon_{\perp}>0$, то есть, в структуре реализуется гиперболический режим дисперсии. На рис. 3, a также представлен спектр поглощения структуры при малом угле падения $12^{\circ}$ (сплошная линия). Видно усиление поглощения в точках ENP и $\mathrm{ENZ}$, что связано с диссипацией энергии падающей световой волны при резонансном возбуждении колебаний электронного газа. На рис. $3, b$ представлены рассчитанные частотно-угловые спектры пропускания исследуемой структуры, демонстрирующие наличие двух минимумов, соответствующих возбуждению указанных выше резонансов.

Небольшие отличия расчетных результатов от экспериментальных можно объяснить тем, что характери- 

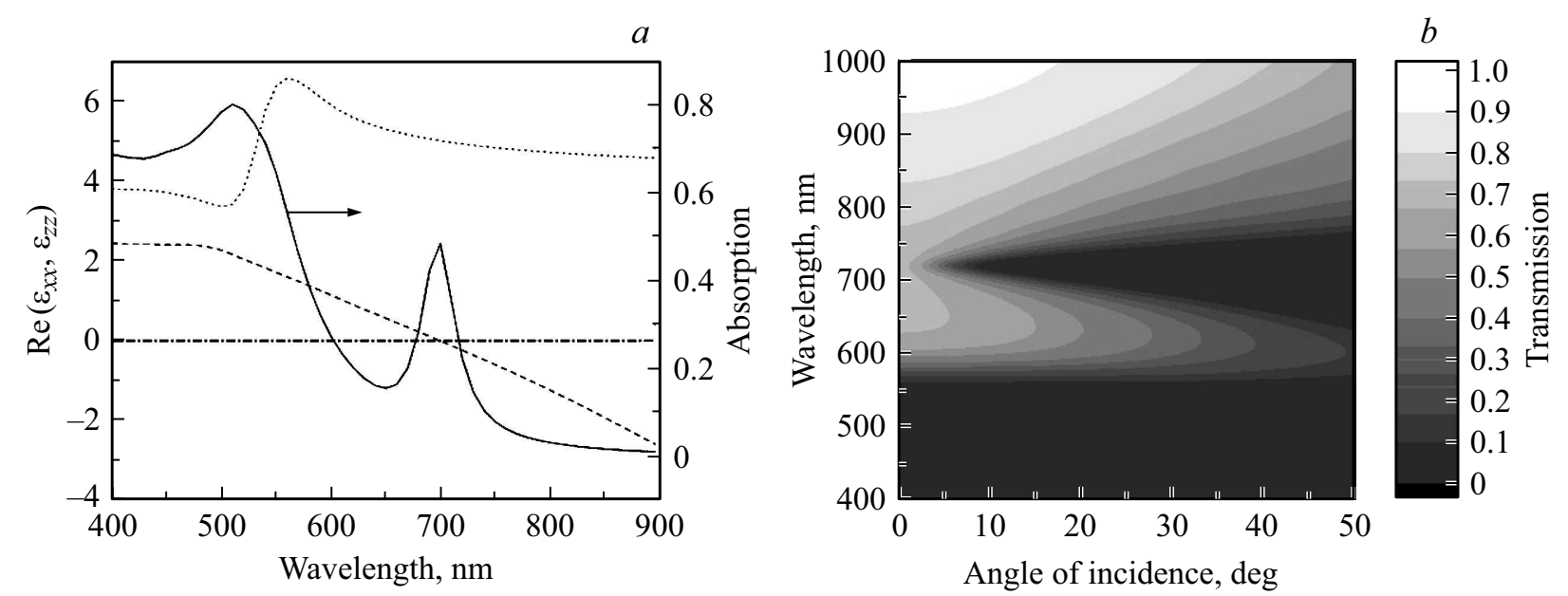

Рис. 3. Результаты численного моделирования: $a-$ компоненты диэлектрической проницаемости $\varepsilon_{\perp}=\varepsilon_{x x}$ (пунктирная линия) и $\varepsilon_{\|}=\varepsilon_{z z}$ (штриховая линия), и поглощения (сплошная линия); (b) - частотно-угловой спектр. Данные приведены для массива золотых наностержней с диаметром 30 и длиной $200 \mathrm{~nm}$. Степень заполнения пространства металлом $17 \%$.

стики электроосажденного золота могут немного отличаться от свойств монокристалла Аи. Детальное исследование состава и структуры наностержней выходит за рамки данной работы и будет выполнено в дальнейшем. Следует также отметить, что модель эффективной среды справедлива, когда длина волны света много больше размеров включений, однако в гиперболическом режиме, когда неограниченно возрастает волновой вектор, а длина волны уменьшается, данное условие не выполняется. Тем не менее, в представленном случае, как и во многих других работах по гиперболическим материалам $[7,16]$, расчеты в рамках модели эффективной среды достаточно хорошо согласуются с экспериментальными данными.

\section{2. Оптический отклик композитных наностержней $\mathrm{Au} / \mathrm{Ni}$}

На рис. 4 представлен спектр пропускания массива композитных наностержней $\mathrm{Au} / \mathrm{Ni}$ с объемной долей металла в заполненной части диэлектрической матрицы $10 \%$, в котором поверх наностержней золота (с длиной $200 \mathrm{~nm}$ ) был осажден никель, длина стержней которого составила около $500 \mathrm{~nm}$. Наличие никеля приводит к значительному снижению коэффициента пропускания структуры, поэтому для стержней $\mathrm{Au} / \mathrm{Ni}$ измерения были выполнены в геометрии на отражение.

Как видно из рис. 4, минимум пропускания такой структуры смещен в красноволновую область спектра и наблюдается при длине волны $800 \mathrm{~nm}$. Согласно выполненным расчетам, такой сдвиг резонанса по сравнению с рассмотренным выше образцом наностержней $\mathrm{Au}$ обусловлен меньшей пористостью структуры и большим аспектным соотношением золотых стержней и соответствует особой точке ENZ, $\varepsilon_{\|} \rightarrow 0$. Обнаружено, что вблизи этой особенности для геометрии экваториального магнитооптического эффекта Керра наблюдается

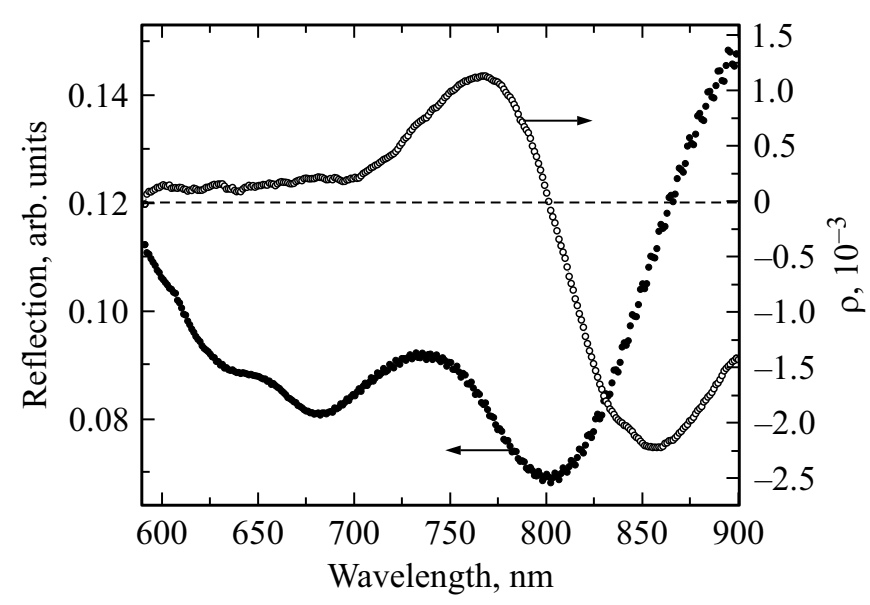

Pис. 4. Спектр коэффициента отражения (сплошные символы) и экваториального магнитооптического эффекта Керра (пустые символы) для образца массива наностержней $\mathrm{Au} / \mathrm{Ni}$ в матрице анодного оксида алюминия. Угол падения равен $45^{\circ}$.

значительная модуляция магнитного контраста (рис. 4, незаполненные символы), причем абсолютная величина $\rho$ возрастает более чем на порядок и достигает максимального значения $\approx 2.2 \cdot 10^{-3}$. Это приблизительно на порядок по величине превышает величину магнитооптического эффекта вдали от резонанса. Наблюдающаяся смена знака магнитного контраста (и прохождение его через нулевое значение) (рис. 4) указывает на то, что намагниченность структуры приводит к нечетному спектральному сдвигу резонансного условия ENZ.

\section{5. Заключение}

Таким образом, в работе методом темплатного электроосаждения в матрицы пористого оксида алюминия 
изготовлены два типа массивов наностержней со структурой золото и золото/никель, и изучены особенности их линейных оптических и магнитооптических спектров. Показано, что в исследованных структурах наблюдаются два вида резонансных особенностей, соответствующих, согласно выполненным расчетам, полюсу диэлектрической проницаемости $\varepsilon_{\perp} \rightarrow \infty(\mathrm{ENP})$ и нулевому значению компоненты $\varepsilon_{\|} \rightarrow 0$ (ENZ). Обнаружено, что для массива наностержней со структурой $\mathrm{Au} / \mathrm{Ni}$ в окрестности особенности ENZ наблюдается смена знака магнитного контраста и значительная модуляция его амплитуды, что является проявлением магнитоиндуцированного сдвига дисперсионной зависимости данного нанокомпозитного материала.

\section{Список литературы}

[1] A. Alu, M.G. Silveirinha, A. Salandrino, N. Engheta. Phys. Rev. B 75, 155410 (2007).

[2] A. Poddubny, I. Iorsh, P. Belov, Yu. Kivshar. Nature Photon. 7, 948 (2013).

[3] P. Shekhar, J. Atkinson, Z. Jacob. Nanoconvergence 1, 14, 1 (2014).

[4] L. Ferrari, Ch. Wu, D. Lepage, X. Zhang, Zh. Liu. Progr. Quantum Electron. 40, 1 (2015).

[5] А.В. Щелокова, П.В. Капитанова, П.А. Белов. Научно-техн. вестн. информ. технологий, механики и оптики 2, 90, 23 (2014).

[6] X. Ni, G.V. Naik, A.V. Kildishev, Y. Barnakov, A. Boltasseva, V.M. Shalaev. Appl. Phys. B 103, (2011).

[7] A.L. Cortes, W. Newman, S. Molesky, Z. Jacob. J. Opt. 14, 6, 129501 (2014).

[8] S. Habouti, M. Matefi-Tempfli, C.-H. Solterbeck, M. Es-Souni, S. Matefi-Tempfli, M. Es-Souni. J. Mater. Chem. 21, 6269 (2011).

[9] L. Ferrari, J. Stephen, Th. Smalley, Y. Fainman, Zh. Liu. Nanoscale 9, 9034 (2017).

[10] K.V. Sreekanth, M. ElKabbash, Y. Alapan, E. Ilker, M. Hinczewski, U.A. Gurkan, G. Strangi. EPJ Appl. Metamater. 4, 1 (2017).

[11] A.V. Kabashin, P. Evans, S. Pastkovsky, W. Hendren, G.A. Wurtz, R. Atkinson, R. Pollard, V.A. Podolskiy, A.V. Zayats. Nature Mater. 8, 867 (2009).

[12] T. Li, V. Nagal, D.H. Gracias, J.B. Khurgin. Opt. Express 25, 12, 13588 (2017).

[13] N. Vasilantonakis, M.E. Nasir, W. Dickson, G.A. Wurtz, A.V. Zayats. Laser Photon. Rev. 9, 3, 345 (2015).

[14] G. Marino, P. Segovia, A.V. Krasavin, P. Ginzburg, N. Olivier, G.A. Wurtz, A.V. Zayats. Laser Photon. Rev. 1700189, 1 (2018).

[15] C.R. Simovski, P.A. Belov, A.V. Atrashchenko, Y.S. Kivshar. Adv. Mater. 24, 4229 (2012).

[16] R. Atkinson, W.R. Hendren, G.A. Wurtz, W. Dickson, A.V. Zayats, P. Evans, R.J. Pollard. Phys. Rev. B 73, 235402 (2006).

[17] P. Evans, W.R. Hendren, R. Atkinson, G.A. Wurtz, W. Dickson, A.V. Zayats, R.J. Pollard. Nanotechnology 17, 5746 (2006).
[18] G.A. Wurtz, W. Dickson, D. O’Connor, R. Atkinson, W. Hendren, P. Evans, R. Pollard, A.V. Zayats. Opt. Express 16, 10, 7460 (2008).

[19] W. Li, Zh. Liu, X. Zhang, X. Jiang. Appl. Phys. Lett. 100, 161108 (2012).

[20] M.S. Mirmoosa, S.Yu. Kosulnikov, C.R. Simovski. Phys. Rev. B 94, 075138 (2016).

[21] S.S. Kruk, Z.J. Wong, E. Pshenay-Severin, K. O’Brien, D.N. Neshev, Y.S. Kivshar, X. Zhang. Nature Commun. 7, 11329 (2016).

[22] V.L. Krutyanskiy, I.A. Kolmychek, E.A. Gan'shina, T.V. Murzina, P. Evans, R. Pollard, A.A. Stashkevich, G.A. Wurtz, A.V. Zayats. Phys. Rev. B 87, 035116 (2013).

[23] V.I. Belotelov, I.A. Akimov, M. Pohl, V.A. Kotov, S. Kasture, A.S. Vengurlekar, A.V. Gopal, D.R. Yakovlev, A.K. Zvezdin, M. Bayer. Nature Nanotechnology 6, 370 (2011).

[24] J.B. Gonzalez-Diaz, A. Garcia-Martin, J.M. Garcia-Martin, A. Cebollada, G. Armelles, B. Sepulveda, Y. Alaverdyan, M. Kall. Small 4, 2, 202 (2008).

[25] A.L. Chekhov, V.L. Krutyanskiy, A.N. Shaimanov, A.I. Stognij, T.V. Murzina. Opt. Express 22, 15, 17764 (2014).

[26] И.А. Колмычек, А.Н. Шайманов, А.В. Барышев, Т.В. Мурзина. Письма в ЖЭТФ 102, 1, 50 (2015).

[27] А.К. Звездин, В.А. Котов. Магнитооптика тонких пленок. Наука, М. (1988). 192 с.

[28] E.D. Palik. Handbook of Optical Constants of Solids. Elsevier (2012). $804 \mathrm{c}$.

Редактор Е.Ю. Флегонтова 